\title{
Rein tension during canter
}

\author{
A. Egenvall ${ }^{1 *}$, M. Eisersiö ${ }^{1}$, M. Rhodin ${ }^{1}$, R. van Weeren ${ }^{2}$ and L. Roepstorff ${ }^{3}$ \\ 'Department of Clinical Sciences, Faculty of Veterinary Medicine and Animal Husbandry, Swedish University of Agricultural Sciences, P.O. \\ Box 7054, 75007 Uppsala, Sweden; ${ }^{2}$ Department of Equine Sciences, Faculty of Veterinary Medicine, Utrecht University, Yalelaan 114, 3584 \\ CM Utrecht, the Netherlands; ${ }^{3}$ Department of Anatomy, Physiology and Biochemistry, Unit of Equine Studies, Faculty of Veterinary Medicine \\ and Animal Husbandry, Swedish University of Agricultural Sciences, P.O. Box 7046, 75007 Uppsala, Sweden; agneta.egenvall@slu.se
}

Received: 27 January 2015 / Accepted: 16 March 2015

RESEARCH ARTICLE

(c) 2015 Wageningen Academic Publishers

\begin{abstract}
Riders generally use reins as a means for communication with the horse. At present, the signalling pattern is poorly understood. The aim of this study was to illustrate and analyse the rein tension patterns in a number of rider/horse combinations across a variety of exercises in the canter gait. Our hypothesis was that some riders will follow the movement of the horse more closely than others. Data were collected from eight professional riders riding each three (in one case two) horses that were familiar to them in canter. Horses were instrumented with rein tension meters logged by inertial measurement unit technique (IMU). Inside and outside rein tension data were synchronised with the gait using the vertical acceleration IMU-signal at the poll. Stride-split data (0-100 percentages) were analysed using mixed models technique to elucidate the inside/outside and stride percentage interaction, taking into account the exercises performed. In general, tension was maximal just before the beginning of vertical stance, as defined by the maximal acceleration of the head, with the release closer to the suspension phase. The release was significantly more marked on the outside rein, but between riders and horses the pattern varied substantially. In total $26 \%$ of the variation was represented by riders and $21 \%$ by the horses. On average there were significant inside/outside rein differences, but at the same time in some horse/rider combinations these differences did not exist.
\end{abstract}

Keywords: rein tension, horses, equine, inertial measurement unit, variation, mixed model

\section{Introduction}

In horseback riding, various signals are used to communicate with the horse. Most of these rely on the exertion of pressure, for instance with 'the legs, the hands, the weight of the rider' (Decarpentry, 1949; McGreevy, 2007). For the communication via the hand, riders have, since ancient times, used reins attached to a bit in the horse's mouth (Warren-Smith et al., 2007; Xenophon, 350 $\mathrm{BC})$. However, despite the fact that this technique has been used for millennia, there is still lack of understanding concerning the exact effects of the reins. Recent studies indicate that the rider has a large influence on the health and well-being of the horse (Egenvall et al., 2013). There are various ways how this influence is exerted, e.g. through the general training strategy (Lönnell et al., 2014; Murray et al., 2010) and the structure of the riding sessions (Eisersiö et al.,
2015). The usage by the rider of cues, such as rein tension, aggravated or not by the use of special bits, is a determinant too (McGreevy, 2007). An often-described goal in dressage is to improve hindlimb activity and self-carriage (GNEF, 1997) (which is equitation terminology for a position that could in biomechanical terms be described as a relatively high carriage of the poll, the nose line perpendicular to the surface and a small weight shift to the hind quarters (Weishaupt et al., 2009) and which is maintained without continuous support by the rider's aids). The position is achieved by a concerted action of the rider's seat, legs and hands, the last aids affecting mainly the head at the bitmouth interface (Eisersiö et al., 2013).

The canter is a three- or four-beat gait. At higher velocities canter will be four-beat when the leading hindlimb will land before the trailing forelimb. In left lead canter the trailing 
right hindlimb touches the ground first, the left hindlimb and right forelimb next, and the leading left forelimb last. The order of the limbs is reversed in right lead canter. The left hindlimb and right forelimb are often synchronous at slower speeds of canter, ranging from 3-5 m/s (Clayton, 1994), making this type of canter a three-beat gait. After the leading forelimb leaves the ground, a suspension phase follows. This phase has been estimated to last from $5 \%$ of total stride time in working canter $(3.9 \mathrm{~m} / \mathrm{s})$ to $54 \%$ in medium canter $(4.9 \mathrm{~m} / \mathrm{s})$ (Clayton, 1994). The trunk of the horse rotates around its transverse axis and the back, particularly the lumbosacral joint, has the largest range of motion in flexion-extension of all gaits in canter (Faber et al., 2001). To ride harmoniously the rider must follow the movements evoked by this gait as closely as possible (e.g. without a large time lag between the motion pattern of the horse and the corresponding motion pattern of the rider).

Most studies on rein tension have been performed in walk and/or trot, e.g. Warren-Smith et al. (2007) and Heleski et al. (2009). Studies on canter have been performed using few horses and riders (Clayton et al., 2003; Kuhnke et al., 2010). There are few studies regarding rein tension in relation to the stride cycle and most of these have been carried out in trot. Clayton et al. (2003) showed rein tension from two strides of canter with a single rider demonstrating one distinct spike during each stride, which was reported to coincide with the time when the horse was supported by a diagonal pair of limbs. At trot, in un-mounted horses or horses ridden in a free head and neck position, the largest rein tension peaks were found at midstance (Clayton $e t$ al., 2011; Eisersiö et al., 2013). This was in contrast to the situation found in three horses ridden in sitting trot with the nose line along the vertical, where the largest rein tension peaks were generally found in the suspension phase (Eisersiö et al., 2013).

Rein tension may vary within and between different conditions: riding sessions, riders and horses (König von Borstel and Glissman, 2014), reins, gaits and exercises (Warren-Smith et al., 2007), and strides (Clayton et al., 2003, 2011; Eisersiö et al., 2013). Canter is an asymmetrical gait with large differences in motion pattern of the horse between the stance and the swing phase and it is also the gait where rein tension has been found to be highest (Warren-Smith et al., 2007). The major aim of this study was to illustrate and analyse the rein tension patterns in a number of rider/horse combinations across a variety of exercises in the canter gait. Our hypothesis was that some riders would follow the movement of the horse more closely than others. Most likely, this higher degree of synchronicity between riders and horses will lead to more consistency in rein tension. Differences in ability to follow, or determined actions during riding, could for example lead to left/right rein tension differences, or to larger differences between the peaks and lows in rein tension that are related to the phase of the stride. Elucidation of the specific, individual tension patterns throughout the stride cycle at canter may be of potential use in the training of riders and may be of benefit in aiding riders to modify the use of the reins when this is believed to be suboptimal.

\section{Materials and methods}

\section{Riders and horses}

The study was observational in character with data collected from regular training sessions of eight professional riders (mean \pm standard deviation; height $173 \pm 6 \mathrm{~cm}$ and weight $65.5 \pm 10 \mathrm{~kg}$ ), who each rode three horses that were familiar to them $(\mathrm{n}=24)$. The riders all worked in the horse industry as riding instructors (with the exception of one, who was only 14 years old, but was training horses in a professional enterprise) and/or horse trainers on various levels. Two riders competed at advanced level (as classified in the national Swedish system), five at intermediate level and two at basic level. Upon request, one rider indicated to be left-handed, the others were right-handed. The horses were between 4 and 27 years old, median 8 years. There were 14 geldings, 9 mares and one stallion. Withers height ranged from 135-180 cm, median $165 \mathrm{~cm}$. Most horses were warmbloods, including Swedish warmbloods ( $\mathrm{n}=13)$, Dutch warmbloods ( $\mathrm{n}=2$ ), one Trakehner, one Latvian warmblood and one Danish warmblood. Breeds also included one of each of the following: Lusitano, Pura Raza Española (Andalusian), Friesian, riding pony, mixed breed and unknown. The educational level of the horse was stated by the riders as: basic $(n=12)$, young horse $(n=3)$, medium $(n=5)$ and advanced $(n=4)$. Advanced horses had competed at Prix St. George, Intermediaire or Grand Prix level; basic horses had entered low-level competitions only and medium horses were in between. Young horses had been ridden for less than a year and had not competed.

Each rider had regularly trained his/her 'own' horses for between one month and 22 years, median 24 months. Most riders had trained their horses exclusively, but three horses (with one rider) were riding school horses that had also been ridden by others. All horses wore their own saddle and bridle with a snaffle bit (Eisersiö et al., 2015). Horse laterality was investigated by asking the riders to which side the horses used to bend most easily.

\section{Equipment}

Data collection took place at each horse's own yard (eight different locations) in arenas located either outdoor $(n=4$ riders, gravel-based, the smallest $23 \times 62 \mathrm{~m}$ and the largest $40 \times 80 \mathrm{~m}$ ) or indoor ( $\mathrm{n}=4$ riders, 2 sand-fibre arenas and 2 sand-wood chip arenas, the smallest $20 \times 50 \mathrm{~m}$ and the largest $24 \times 62 \mathrm{~m}$ ). Each horse was fitted with a custommade rein tension meter $(128 \mathrm{~Hz})$, measuring range 0-500 
$\mathrm{N}$, resolution $0.11 \mathrm{~N}$, fastened on leather reins. A cable from each tension meter ran forwards along each of the reins and up along the side piece of the bridle, passing behind the horse's ear to an Inertial Measurement Unit (IMU, $\mathrm{x}$-io Technologies Limited, Bristol, UK) attached right below the browband of the bridle using a custom made Velcro browband. The rein tension meters, for each rein separately, were calibrated before the riding sessions started by suspending 13 known weights between 0-20 $\mathrm{kg}$. Further details on the rein tension meter can be found elsewhere (Eisersiö, 2013). All equipment was fitted on the horse in the riding arena, which took approximately $10 \mathrm{~min}$, including synchronisation (see below) of the equipment. Video recordings (Canon Legria HF200, 25 Hz; Canon, Tokyo, Japan) were made of the entire riding session from the middle of one of the long sides of the arena. All horses were free from lameness according to a veterinarian, who visually evaluated the videos of the horses.

\section{Data collection and management}

The riders were asked to follow their normal routine with each horse for flatwork/dressage and to ride in all gaits (walk, trot and canter). The median duration of the riding sessions was $31 \mathrm{~min}$ (range 23-44 $\mathrm{min}$ ) and the median proportion of the riding session spent in each gait was for walk $38 \%\left(25^{\text {th }}\right.$ percentile $/ 75^{\text {th }}$ percentile (P25/P75): 25/51\%), trot 39\% (P25/P75: 32/46\%), left lead canter 8\% (P25/P75: 4/14\% (23 horses)) and for right lead canter 9\% (P25/P75: 6/15\%) (Eisersiö et al., 2015). The whole riding arena was used for the exercises and the duration of the riding session was determined by the rider.

After the rider had mounted, and before dismounting at the end, the rein tension meter was synchronised with the video recordings by pulling on the right tension meter five times twice in a row while counting out loud in front of the camera.

One investigator (ME) scrutinised the videos and categorised the data as described earlier (Eisersiö et al., 2015). In short, the main categories were gait (walk, trot, left lead canter, right lead canter), rider's seat (sitting, light seat, posting), corners and turns (corner left/right, turn left/right), lateral movements (half-pass to the left/ right, shoulder-in left/right, leg-yield left/right) or riding in collection or lengthening (trot, canter). The evaluatorcategorised video data (mainly gait) were verified by comparing them to head acceleration data and main categorisations by a second researcher (AE). Rein tension data were downloaded to a personal computer and analysed in Matlab (Matlab; MathWorks Inc., Natick, MA, USA). Using custom-written scripts, data from canter sequences were divided into sequences each containing data from one stride, i.e. the data were 'stride-split' using the most vertical acceleration signal from the poll. The strides were split on the largest positive acceleration in the time lapse between vertical midstance of the leading forelimb in one stride to the same point in the next stride. Time-normalised rein data (0-100\%) were constructed using stride-split data. The nose angle range of motion, as well as whether the nose line was moving backwards (or 'in', i.e. towards a position behind the vertical) or forward (or 'out', i.e. towards a position before the vertical) relative to the frame of the horse, was determined from Euler angles, derived from the gyroscopic IMU signal.

\section{Statistical modelling}

The outcome was rein tension in the left and right rein during canter and data on reins were combined in the same model. Dependent data were time-normalised stride means (one series in one horse $=$ one normalised stride) of rein tension and were checked for normality (i.e. means and medians were close, the standard deviations judged as small, and skewness and kurtosis close to zero), or otherwise suitably transformed.

Fixed effects modelled over the stride, i.e. varied over a normalised mean stride, were stride percentage $(0-100 \%)$, and whether the nose angle increased or decreased. The rest of the fixed effects were measured at the trial level, i.e. were constant during each normalised mean stride. Nose angle ROM was entered as a fixed effect, i.e. the difference between the maximum and minimum nose angle in degrees. This variable was first tested as a dummy variable to check linearity versus rein tension and split up as $<15$ degrees, $\geq 15<20$ degrees, $\geq 20<25$ degrees, $\geq 25<30$ degrees and $\geq 30$ degrees. Other fixed effect variables were whether the horse-rider combination was turning (left/right or baseline not turning), passed through a corner (left/right or baseline not passing through corners), performed lateral movements (half-pass left/right direction [moving on three or four tracks to the left/right neck bent in the direction of travel] or baseline no lateral movements) or was riding in collection (defined as that the horse is taking shorter strides with a rounded croup, neck high and flexion at the poll) or lengthening (defined as that the horse is extending the length of the strides). The activity was also categorised according to seat (sitting/light seat). Educational level of the horse (advanced, medium, young horse and basic) was included as a fixed effect. Inside/outside rein, defined as whether the rein was on the same (inside) or opposite side (outside) as the leading limb in the canter [note that this definition may not always be congruent with equitation terminology for inside/outside rein], was forced in as a fixed effect. The two-way interaction between rein and stride percentage was tested.

There were four random effects: horse-side, rider, horse and category within horse-side. The horse-side effect essentially modelled inside/outside reins in the random effect. The 
percentage of the variation contributed by horse and rider was estimated. The rein and stride percentage interaction was scrutinised and further analysed and stratified by horse to look at the pattern of this interaction within horse. The random effects were reduced to only trial within horse-side for the horse models, and fixed effects with one category were successively removed. In an effort to examine whether horse laterality had any influence on rein tension this variable was tested in the final model (in four variants because two horses were ambiguously categorised, see first paragraph in results). Also, using the whole dataset a threeway interaction term between canter, stride percentage and rein was tested for illustration of inside-outside rein effects in the two canter leads. Models were reduced based on the type III sums of squares. The correlation structure was variance component (SAS Institute Inc., Cary, NC, USA). Pair-wise comparisons were done where interactions were involved. Variables were kept if $P<0.05$, but pair-wise comparisons were only considered significant if $P<0.001$ (in the graphs if $P<0.0001$ ).

\section{Results}

\section{Descriptive data}

Data from 23 horses were included as in one Swedish warmblood gelding (of young horse educational level) a problem was encountered when splitting the data by stride. Seven horses were classified as left lateralised (easier to bend to the left), 15 horses as right lateralised. Two horses could not be classified unambiguously. One of these was equally lateralised left and right, and one horse was considered right lateralised at trot and left lateralised at canter.
Table 1 shows the distribution of the normalised rein data per category (i.e. turn, corner, etc., see columns 3-7). For each category and horse there were between 2 and 676, median 40/47 strides for left/ right canter. With three riders the left rein tension was only partly captured due to equipment failure.

The resulting dataset contained 36,764 data points/364 normalised strides (101 data points per rein). The nose angle ROM varied across horses from 8-38 degrees and by category from 16-37 degrees. This was distributed as $<15$ degrees, 2,828 observations/28 strides, $\geq 15<20$ degrees 7,070 observations/70 strides, $\geq 20<25$ degrees 9,393 observations/93 strides, $\geq 25<30$ degrees 9,191 observations/91 strides, $\geq 30$ degrees 8,282 observations/82 strides. In 11,675 (45\%) of the observations the nose was moving in, and in 20,089 (55\%) it was moving out. We were not able to make sure that the absolute angle was correctly measured in all cases, and therefore only included data on the ROM and whether the nose angle increased or decreased. Figure 1 shows an example of normalised raw (calibrated) canter data with the stride-split indicated by bars. Supplementary Figure S1A-B shows examples of normalised mean strides.

\section{The models}

Rein tension was deemed best as logarithm-transformed. In the logarithm-transformed format the descriptive statistics were: mean 3.1 , std 0.7 , median $3.2,1^{\text {st }}$ percentile $1.4,99^{\text {th }}$ percentile 4.5, skewness -0.5, kurtosis 1.0. All main effects had a $P$-value of $<0.05$ (Table 2). Controlling for the stride cycle and its interaction with the inside/outside rein and

Table 1. Basic descriptive rein tension statistics and data by category. Data are from eight riders riding 23 horses in dressage canter of their own choice.

\begin{tabular}{|c|c|c|c|c|c|c|c|c|c|c|c|c|c|}
\hline \multirow{2}{*}{$\begin{array}{l}\text { Canter } \\
\text { direction }^{1}\end{array}$} & \multirow[t]{2}{*}{ Rein } & \multicolumn{5}{|c|}{ Combined category ${ }^{1}$} & \multirow{2}{*}{$\begin{array}{l}\text { No. } \\
\text { horses }^{2}\end{array}$} & \multirow{2}{*}{$\begin{array}{l}\text { Mean no. } \\
\text { strides }^{2}\end{array}$} & \multicolumn{5}{|c|}{ Rein tension $(n)^{3}$} \\
\hline & & Turns & Corners & $\begin{array}{l}\text { Lateral } \\
\text { movements }\end{array}$ & Seat & $\begin{array}{l}\text { Collection/ } \\
\text { lengthening }\end{array}$ & & & mean & SD & median & $\min$ & $\max$ \\
\hline $\mathrm{L}$ & inside & $\mathrm{BL}$ & $\mathrm{BL}$ & half-pass L & sitting & $\mathrm{BL}$ & 8 & 32 & 39 & 15 & 39 & 10 & 74 \\
\hline L & inside & $\mathrm{BL}$ & $\mathrm{BL}$ & $\mathrm{BL}$ & light & $\mathrm{BL}$ & 10 & 29 & 19 & 9 & 20 & 3 & 45 \\
\hline L & inside & $\mathrm{BL}$ & $\mathrm{BL}$ & $\mathrm{BL}$ & light & lengthen & 1 & 12 & 54 & 7 & 55 & 42 & 65 \\
\hline $\mathrm{L}$ & inside & $\mathrm{BL}$ & $\mathrm{BL}$ & $\mathrm{BL}$ & sitting & $\mathrm{BL}$ & 16 & 71 & 29 & 13 & 27 & 5 & 70 \\
\hline $\mathrm{L}$ & inside & $\mathrm{BL}$ & $\mathrm{BL}$ & $\mathrm{BL}$ & sitting & collection & 1 & 24 & 18 & 11 & 16 & 4 & 35 \\
\hline $\mathrm{L}$ & inside & $\mathrm{BL}$ & $\mathrm{BL}$ & $\mathrm{BL}$ & sitting & lengthen & 3 & 8 & 32 & 23 & 25 & 5 & 97 \\
\hline L & inside & $\mathrm{BL}$ & L corner & $\mathrm{BL}$ & light & $\mathrm{BL}$ & 4 & 7 & 21 & 12 & 22 & 5 & 51 \\
\hline $\mathrm{L}$ & inside & $\mathrm{BL}$ & Lcorner & $\mathrm{BL}$ & sitting & $\mathrm{BL}$ & 6 & 17 & 26 & 14 & 23 & 5 & 57 \\
\hline $\mathrm{L}$ & inside & turn $\mathrm{L}$ & $\mathrm{BL}$ & half-pass L & sitting & $\mathrm{BL}$ & 3 & 24 & 30 & 8 & 32 & 10 & 46 \\
\hline $\mathrm{L}$ & inside & turn $\mathrm{L}$ & $\mathrm{BL}$ & $\mathrm{BL}$ & light & $\mathrm{BL}$ & 11 & 58 & 22 & 11 & 20 & 6 & 65 \\
\hline L & inside & turn L & $\mathrm{BL}$ & $\mathrm{BL}$ & sitting & $\mathrm{BL}$ & 17 & 201 & 25 & 12 & 24 & 6 & 58 \\
\hline
\end{tabular}


Table 1. Continued.

\begin{tabular}{|c|c|c|c|c|c|c|c|c|c|c|c|c|c|}
\hline \multirow{2}{*}{$\begin{array}{l}\text { Canter } \\
\text { direction } 1\end{array}$} & \multirow{2}{*}{ Rein } & \multicolumn{5}{|c|}{ Combined category ${ }^{1}$} & \multirow{2}{*}{$\begin{array}{l}\text { No. } \\
\text { horses }\end{array}$} & \multirow{2}{*}{$\begin{array}{l}\text { Mean no. } \\
\text { strides }^{2}\end{array}$} & \multicolumn{5}{|c|}{ Rein tension $(n)^{3}$} \\
\hline & & Turns & Corners & $\begin{array}{l}\text { Lateral } \\
\text { movements }\end{array}$ & Seat & $\begin{array}{l}\text { Collection/ } \\
\text { lengthening }\end{array}$ & & & mean & SD & median & $\min$ & $\max$ \\
\hline $\mathrm{L}$ & inside & turn $\mathrm{L}$ & $B L$ & $B L$ & sitting & collection & 2 & 61 & 29 & 12 & 28 & 8 & 57 \\
\hline $\mathrm{L}$ & inside & turn $\mathrm{R}$ & $B L$ & $\mathrm{BL}$ & sitting & $\mathrm{BL}$ & 5 & 29 & 23 & 11 & 22 & 3 & 53 \\
\hline L & outside & $\mathrm{BL}$ & $B L$ & half-pass L & sitting & $B L$ & 8 & 32 & 42 & 24 & 34 & 10 & 102 \\
\hline L & outside & $B L$ & $B L$ & $\mathrm{BL}$ & light & $\mathrm{BL}$ & 11 & 27 & 18 & 13 & 14 & 4 & 78 \\
\hline L & outside & $B L$ & $B L$ & $B L$ & light & lengthen & 1 & 12 & 58 & 6 & 59 & 46 & 65 \\
\hline $\mathrm{L}$ & outside & $B L$ & $B L$ & $B L$ & sitting & $\mathrm{BL}$ & 17 & 68 & 32 & 21 & 28 & 6 & 104 \\
\hline $\mathrm{L}$ & outside & $B L$ & $B L$ & $B L$ & sitting & collection & 1 & 24 & 27 & 18 & 22 & 7 & 60 \\
\hline L & outside & $B L$ & $\mathrm{BL}$ & $B L$ & sitting & lengthen & 3 & 8 & 39 & 26 & 30 & 8 & 104 \\
\hline L & outside & $B L$ & Lcorner & $\mathrm{BL}$ & light & $\mathrm{BL}$ & 4 & 7 & 14 & 14 & 8 & 2 & 65 \\
\hline $\mathrm{L}$ & outside & $\mathrm{BL}$ & Lcorner & $\mathrm{BL}$ & sitting & $B L$ & 6 & 17 & 32 & 20 & 25 & 4 & 87 \\
\hline L & outside & turn $\mathrm{L}$ & $\mathrm{BL}$ & half-pass L & sitting & $B L$ & 3 & 24 & 31 & 15 & 33 & 10 & 55 \\
\hline L & outside & turn $\mathrm{L}$ & $\mathrm{BL}$ & $\mathrm{BL}$ & light & $B L$ & 12 & 60 & 19 & 16 & 14 & 4 & 81 \\
\hline L & outside & turn $\mathrm{L}$ & $B L$ & $B L$ & sitting & $\mathrm{BL}$ & 18 & 199 & 29 & 19 & 23 & 6 & 99 \\
\hline L & outside & turn $\mathrm{L}$ & $\mathrm{BL}$ & $B L$ & sitting & collection & 2 & 61 & 27 & 18 & 19 & 8 & 65 \\
\hline$L$ & outside & turn $R$ & $\mathrm{BL}$ & $\mathrm{BL}$ & sitting & $\mathrm{BL}$ & 5 & 29 & 28 & 15 & 24 & 9 & 75 \\
\hline $\mathrm{R}$ & inside & $\mathrm{BL}$ & $\mathrm{BL}$ & half-pass $R$ & sitting & $B L$ & 6 & 14 & 54 & 25 & 55 & 12 & 120 \\
\hline$R$ & inside & $B L$ & $\mathrm{BL}$ & $\mathrm{BL}$ & light & $\mathrm{BL}$ & 10 & 28 & 18 & 8 & 18 & 1 & 54 \\
\hline$R$ & inside & $B L$ & $B L$ & $\mathrm{BL}$ & light & lengthen & 1 & 2 & 44 & 14 & 44 & 20 & 68 \\
\hline $\mathrm{R}$ & inside & $\mathrm{BL}$ & $\mathrm{BL}$ & $\mathrm{BL}$ & sitting & $\mathrm{BL}$ & 17 & 65 & 32 & 18 & 28 & 5 & 102 \\
\hline$R$ & inside & $B L$ & $B L$ & $B L$ & sitting & collection & 1 & 21 & 23 & 11 & 23 & 8 & 41 \\
\hline $\mathrm{R}$ & inside & $\mathrm{BL}$ & $\mathrm{BL}$ & $\mathrm{BL}$ & sitting & lengthen & 3 & 7 & 37 & 17 & 36 & 6 & 89 \\
\hline $\mathrm{R}$ & inside & $\mathrm{BL}$ & R corner & $\mathrm{BL}$ & light & $\mathrm{BL}$ & 5 & 8 & 14 & 9 & 14 & 0 & 51 \\
\hline $\mathrm{R}$ & inside & $\mathrm{BL}$ & R corner & $\mathrm{BL}$ & sitting & $B L$ & 10 & 19 & 35 & 17 & 30 & 7 & 89 \\
\hline $\mathrm{R}$ & inside & turn $\mathrm{L}$ & $\mathrm{BL}$ & $\mathrm{BL}$ & sitting & $\mathrm{BL}$ & 5 & 28 & 29 & 18 & 23 & 4 & 70 \\
\hline $\mathrm{R}$ & inside & turn $\mathrm{R}$ & $\mathrm{BL}$ & half-pass $R$ & sitting & $\mathrm{BL}$ & 3 & 37 & 36 & 16 & 32 & 10 & 79 \\
\hline $\mathrm{R}$ & inside & turn $\mathrm{R}$ & $B L$ & $\mathrm{BL}$ & light & $\mathrm{BL}$ & 12 & 82 & 23 & 14 & 20 & 4 & 68 \\
\hline $\mathrm{R}$ & inside & turn $R$ & $B L$ & $\mathrm{BL}$ & sitting & $\mathrm{BL}$ & 18 & 166 & 30 & 16 & 26 & 4 & 92 \\
\hline$R$ & inside & turn $\mathrm{R}$ & $\mathrm{BL}$ & $\mathrm{BL}$ & sitting & collection & 4 & 43 & 31 & 18 & 28 & 3 & 83 \\
\hline $\mathrm{R}$ & outside & $\mathrm{BL}$ & $\mathrm{BL}$ & half-pass $R$ & sitting & $\mathrm{BL}$ & 6 & 14 & 39 & 23 & 33 & 11 & 120 \\
\hline $\mathrm{R}$ & outside & $\mathrm{BL}$ & $\mathrm{BL}$ & $\mathrm{BL}$ & light & $\mathrm{BL}$ & 10 & 28 & 17 & 10 & 15 & 2 & 44 \\
\hline $\mathrm{R}$ & outside & $B L$ & $B L$ & $B L$ & light & lengthen & 1 & 2 & 31 & 8 & 31 & 19 & 49 \\
\hline $\mathrm{R}$ & outside & $\mathrm{BL}$ & $\mathrm{BL}$ & $\mathrm{BL}$ & sitting & $B L$ & 16 & 65 & 30 & 18 & 26 & 7 & 109 \\
\hline $\mathrm{R}$ & outside & $\mathrm{BL}$ & $\mathrm{BL}$ & $\mathrm{BL}$ & sitting & collection & 1 & 21 & 20 & 10 & 20 & 7 & 37 \\
\hline $\mathrm{R}$ & outside & $B L$ & $\mathrm{BL}$ & $\mathrm{BL}$ & sitting & lengthen & 3 & 7 & 34 & 13 & 30 & 13 & 73 \\
\hline$R$ & outside & $B L$ & R corner & $\mathrm{BL}$ & light & $\mathrm{BL}$ & 5 & 8 & 16 & 9 & 14 & 3 & 43 \\
\hline$R$ & outside & $\mathrm{BL}$ & R corner & $\mathrm{BL}$ & sitting & $B L$ & 9 & 18 & 30 & 19 & 28 & 5 & 112 \\
\hline $\mathrm{R}$ & outside & turn $\mathrm{L}$ & $\mathrm{BL}$ & $\mathrm{BL}$ & sitting & $\mathrm{BL}$ & 5 & 28 & 24 & 14 & 22 & 6 & 65 \\
\hline$R$ & outside & turn $R$ & $B L$ & half-pass $R$ & sitting & $B L$ & 3 & 37 & 33 & 18 & 30 & 9 & 74 \\
\hline $\mathrm{R}$ & outside & turn $\mathrm{R}$ & $B L$ & $\mathrm{BL}$ & light & $\mathrm{BL}$ & 11 & 79 & 17 & 13 & 13 & 3 & 77 \\
\hline $\mathrm{R}$ & outside & turn $\mathrm{R}$ & $B L$ & $\mathrm{BL}$ & sitting & $\mathrm{BL}$ & 17 & 166 & 26 & 15 & 23 & 5 & 96 \\
\hline $\mathrm{R}$ & outside & turn $R$ & $\mathrm{BL}$ & $\mathrm{BL}$ & sitting & collection & 4 & 43 & 24 & 20 & 19 & 2 & 81 \\
\hline
\end{tabular}

${ }^{1} \mathrm{~L}=$ left; $\mathrm{R}=$ right; $\mathrm{BL}=$ baseline (activity without turns, corners, lateral movements or collection/lengthening respectively).

2 The number of horses equals the number of normalised strides analysed per category, while mean number of strides shows the number of original strides the normalised strides were based on. In some instances there was data loss on the left rein, for this reason the numbers differ between left and right canter direction.

${ }^{3}$ Rein tension statistics are based on the stride-split data set (total $\mathrm{n}$ data points=36,764); $\mathrm{SD}=$ standard deviation. 


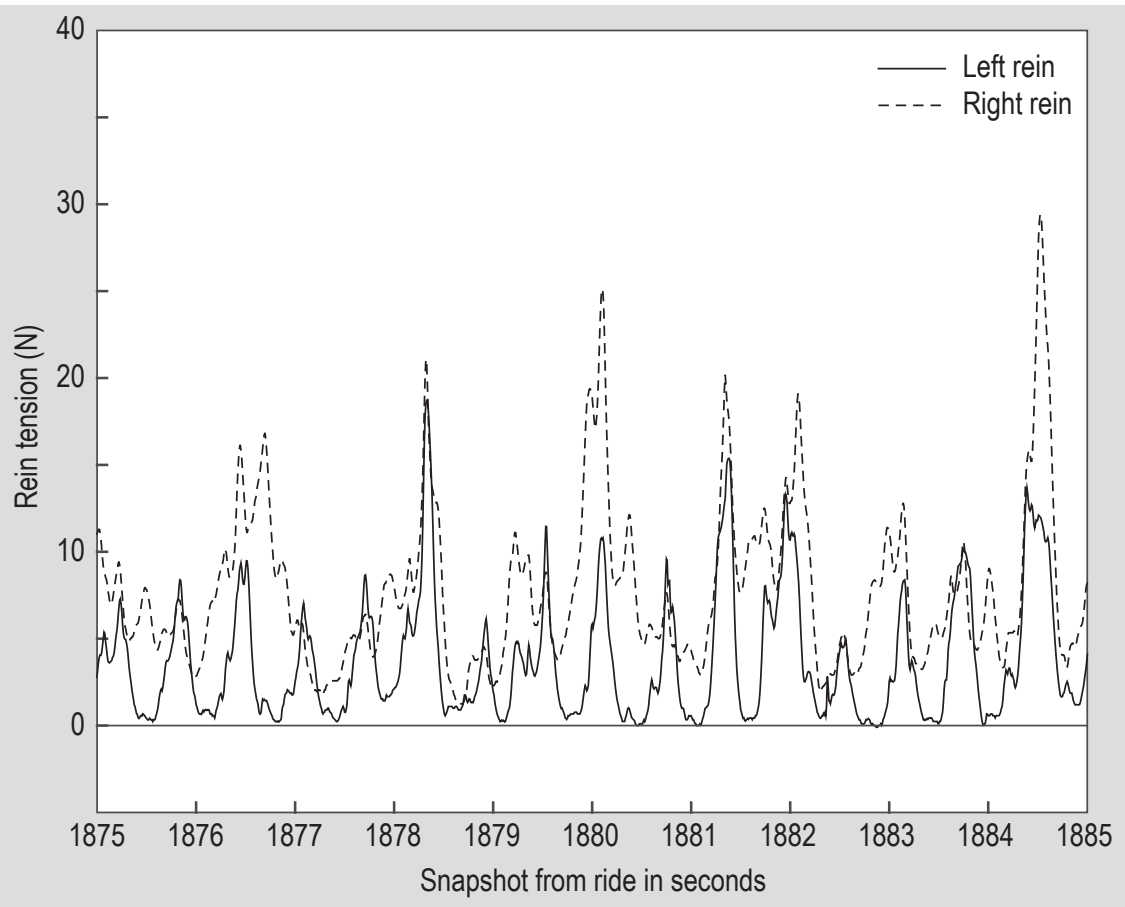

Figure 1. Graph of raw (calibrated) rein tension data at left canter (coded as turn right, sitting), stride-split (black bars) at the highest vertical head acceleration (not shown) in the combination of rider 1 with horse 3 . The lines show tension in the left and right rein respectively.

all other variables in the model, the numerical differences were rather small, the largest difference (where even the confidence intervals did not overlap) was found within horse level, where advanced $(24.2 \mathrm{~N})$ and young horses $(28.7$ $\mathrm{N})$ were associated with a higher tension than basic horses $(13.3 \mathrm{~N})$. Lengthening $(25 \mathrm{~N})$ was associated with a higher rein tension than collection $(15.5 \mathrm{~N})$. Turns, corners, and lateral movements were associated with a lower tension than not performing these exercises and the light seat with less tension than sitting canter (Table 2). Also, if the nose moved forward ('out': $20.2 \mathrm{~N}$ ) the tension was higher than if the nose was moving backwards ('in': $19.7 \mathrm{~N}$ ). This variable was analysed within the stride and 'potentially changing' with every stride percentage, for which reason the seemingly minuscule difference could become significant. A sensitivity analysis was performed omitting the trials with a low number of strides ( $<4$ strides, 21 trials). In this situation, the variable collection/lengthening was no longer significant, but differences between all other variables (both with respect to direction and significance) were preserved (data not shown).

Model least-square mean results of rein tension for the inside and outside rein over the stride cycles are shown in Figure 2. Results are controlled for the variables in the model, i.e. they represent the marginal means over a population balanced in terms of variables and categories within the independent variables. In general, maximum rein tension was found just before or after the beginning of vertical midstance of the leading forelimb, as defined by the acceleration of the head, while maximal release seemed to occur closer to the suspension phase for the inside rein, and somewhat later for the outside rein. The release was more marked on the outside rein, with pressure being significantly lower in both leads at $30 \%$ of the stride. In comparison during stance phase of the leading forelimb there was hardly any difference between the reins. There was a significant difference $(P<0.0001)$ between both canter leads (as depicted in Figure 4), but the graph also showed that the outside rein in left canter had a significantly higher rein tension than the outside rein in right canter, suggesting an effect of rider and/or horse laterality. However, when verifying against horse laterality (modelling horse laterality upon the model in Table 2) no significant impact on the data could be found ( $P>0.7$, data not shown). In the overall model $26 \%$ and $21 \%$ of the variation could be attributed to the riders and the horses respectively.

\section{The effect of the stride cycle relative to the horses}

Data on individual horses are given in Figure 3 and Supplementary Figure S2. In total 0-7 fixed effects were removed from the horse-specific models (median 3). The stride cycle had a large effect on rein tension. This effect was seen for all riders. Maximum rein tension occurred during leading forelimb stance and minimal rein tension occurred closer to the suspension phase of the canter stride. The outside rein invariably recorded lower tension in those cases 
Table 2. Results of rein tension (back-transformed, in $\mathrm{N}$, modelled in logarithm-transformed format) analysis from the multivariable modelling ( 8 riders, 23 horses, 364 normalised strides [in total 36,764 data points]) presenting least-square means from the model. The model also contained the fixed effects of stride percentage $(P<0.0001)$ and its interaction with inside/outside rein $(P<0.0001)$.

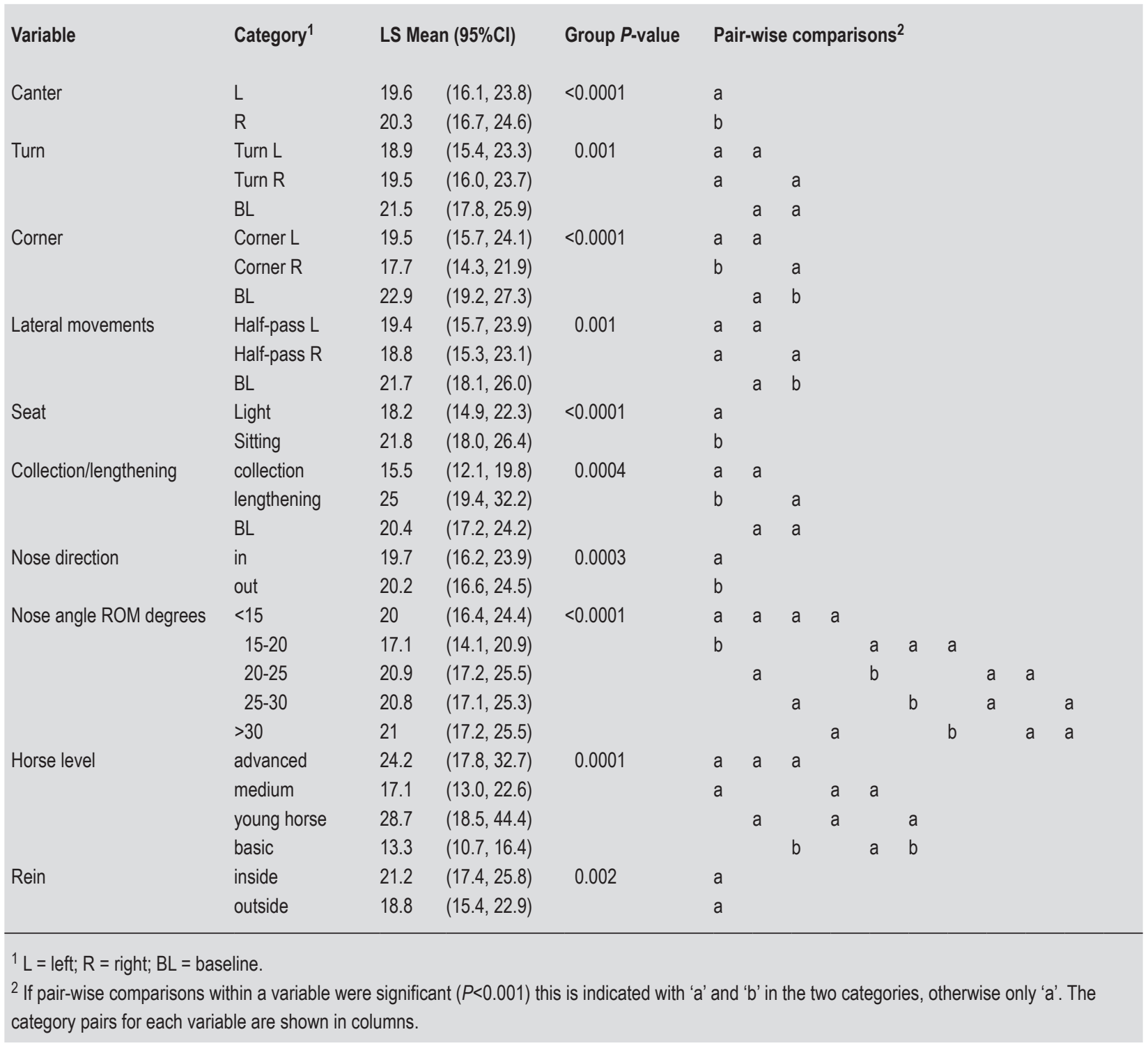

in which minimal rein tension was significantly different between the right and left rein. Figure 3 and Supplementary Figure S2 suggest there were rider-specific differences, which were significant during different parts of the stride cycle, revealing systematic timing differences between riders. Some riders demonstrated a larger phase-shift of the reins compared to others (e.g. rider 8 in Supplementary Figure S2). In one rider (rider 1, Figure 3) the inside and outside rein tensions seemed to follow each other more closely than in other riders, at least in two horses. In contrast, in rider 4. (Figure 3) the difference between inside and outside rein tensions was relatively large during the last part of the stride cycle. Other riders often exhibited a more variable rein tension pattern.

\section{Discussion}

In contrast to what was found by Kuhnke et al. (2010) we found that the horses were consistently 'ridden more on the inside rein', i.e. that tension was on average higher on the inside rein. An obvious reason for this generally higher tension in the inside rein is that this is the rein that is used to give the primary signal when making turns, going through corners, or asking specific exercises, such as shoulder in or half-pass. In all those cases slightly more tension will be exerted on the inside rein while the outside rein yields a little, while still remaining under tension to retain balance. Of course, the situation is more complex than this in real life. For example, it has been shown that horses may support 


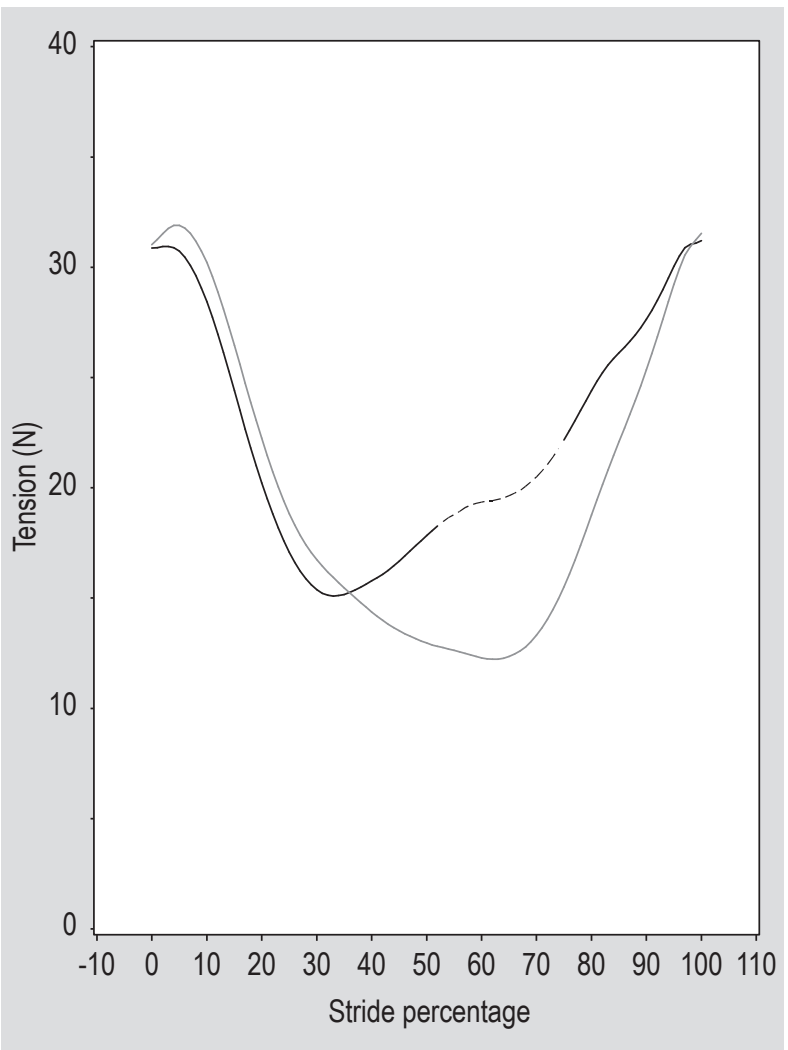

Figure 2. Mean rein tension during the stride cycle for the inside (black) and outside (grey) rein. The part of the stride during which the difference between the inside and outside rein is significant $(P<0.0001)$, is shown as a broken line in the graph representing the inside rein. themselves on the reins while moving, e.g. by detecting the largest rein tension peaks at midstance in unmounted trot (Clayton et al., 2011). When used for signalling, the forces applied to the rein by the hand of the rider are mostly not 'directly' acting in backwards direction, but often will have a more oblique direction when the hand is at the same time displaced in lateral direction. The hand is often moved towards or from the sagittal plane of the horse, often with the intent of lateral displacing, e.g. forequarters relative to the hindquarters, or vice versa, e.g. when turning or performing lateral movements. The situation is further complicated by weight shifts of the rider, when the rider moves, and the ensuing weight-shifting reactions of the horse. It has further been found that, e.g. left-lateralised horses, leaned more on the preferred side (left rein) and avoided contact with the non-preferred side (right rein) (Kuhnke et al., 2010). The rolling motion and lateral asymmetry of canter may be another reason why the inside rein has a greater support/contact. One study compared rider position at first contact of the trailing hindlimb, the diagonal limb pair and the leading forelimb and showed that the rider's trunk was tilted forwards during trailing hindlimb stance and then rotated back towards the vertical during leading forelimb stance and suspension (Lovett et al., 2004). This may influence inside rein tension during the suspension phase when the rider is rotated away from the inside rein and towards the outside (and hence likely to increase tension on the inside rein). It has further been shown that riders did not rotate their upper body symmetrically comparing between left and right canter, but

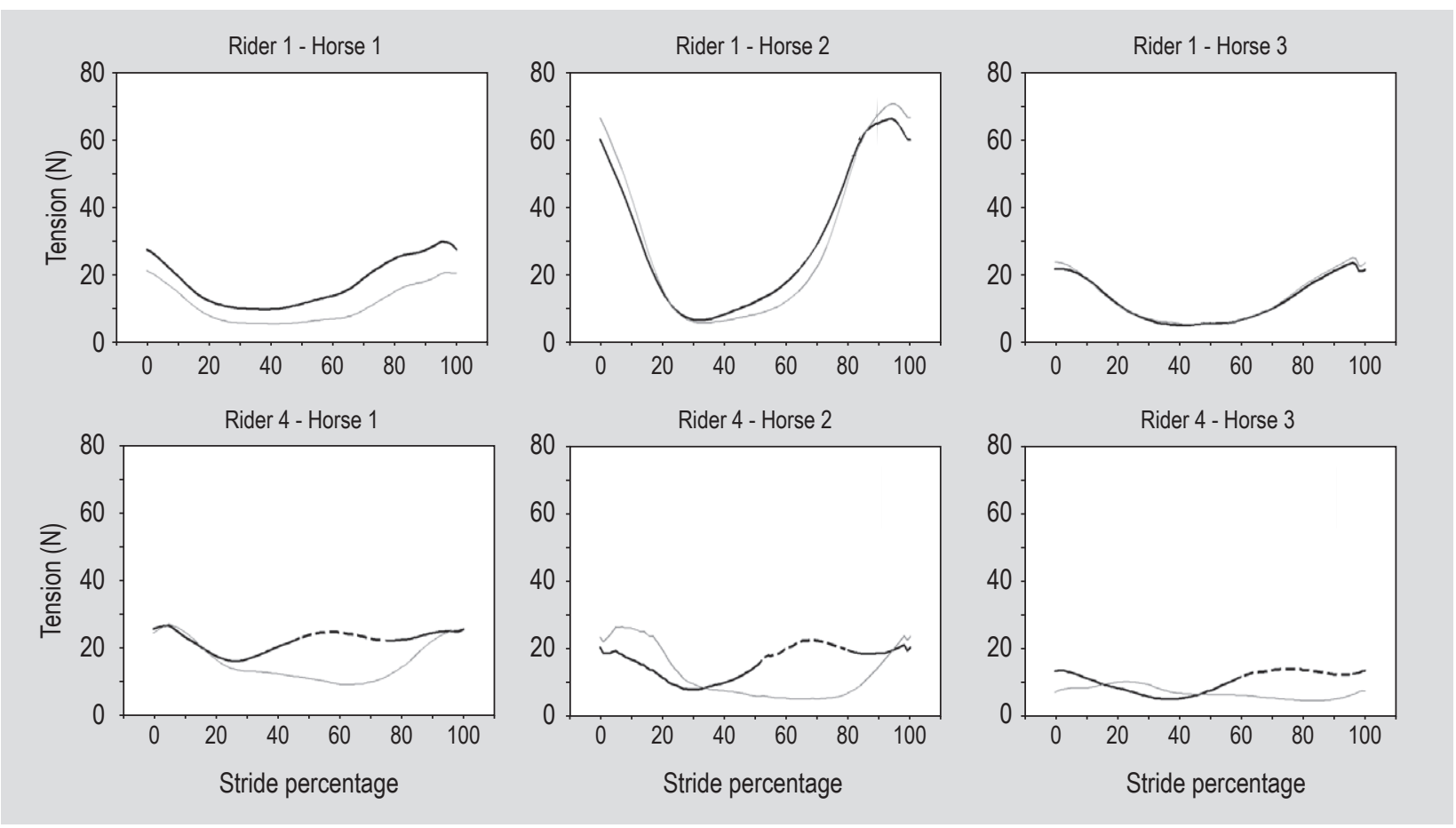

Figure 3. Rein tension during the stride cycle for the inside (black) and outside (grey) rein per horse in rider 1 and 4. Each row indicates one rider. Significant differences $(P<0.0001)$ between the inside and outside rein are shown as broken lines in the inside rein. 


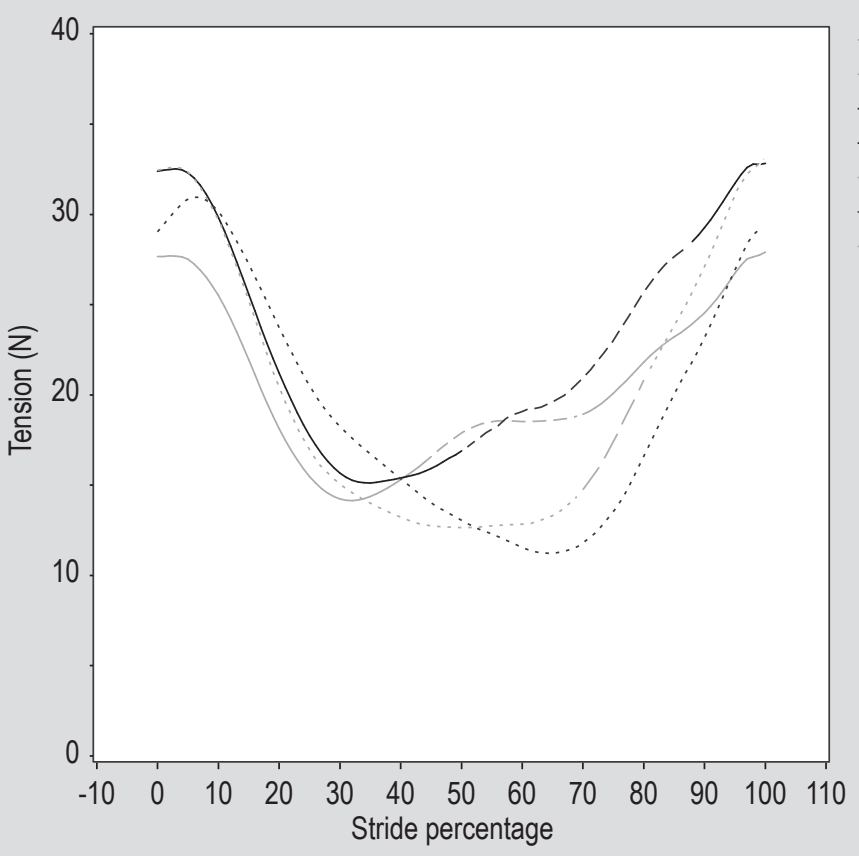

$L$ rein $L$ canter

$R$ rein $L$ canter

$\mathrm{R}$ rein $\mathrm{R}$ canter

$L$ rein $R$ canter

Difference $L / R$ rein in $L$ (grey lines) canter

- - - Difference $L / R$ rein in $R$ (black lines) canter

Difference outside reins (dashed lines)

Figure 4. Back-transformed least square interaction estimates from a three-way interaction between canter (left (L)/right (R)), rein (inside/outside shown as $L / R$ ) and stride percentage $(0-100 \%)$. Significant differences $(P<0.0001)$ between the inside and outside rein in $L$ canter are shown as dashed grey in the inside rein, differences $(P<0.0001)$ between the inside and outside rein in $R$ canter are shown as dashed black in the inside rein and differences $(P<0.0001)$ between the outside reins are shown as long dashed grey in the outside/ $R$ rein in $L$ canter.

were consistently more rotated to the left. The data were interpreted as that riders managed the seat at left canter at more ease than right canter (Symes and Ellis, 2009). In trot it has been shown that the external rotation of the right hip was larger than that of the left hip (Gandy et al., 2014). Whereas these rider asymmetries may indeed have impact on the resulting left and right rein tension, they should, however, not be at the origin of inside/outside rein differences.

Knowledge of how to apply negative reinforcement by manipulation of the reins, with rein tension being relieved as soon as an intended response occurs, is one key for effective training (McGreevy, 2007). At present, it is difficult for trainers to detect how riders are using their hands when teaching riding, but rein tension data may be very helpful here. We therefore hope that the data from this study (that potentially could be used for such a purpose but have not been elaborated as such) may serve as an example how to generate much needed information for riding instruction and will also incite further and more extensive research.

\section{The controlled results from the models}

The modelled fixed effects (except for rein, canter and stride percentage) were a priori included to control these effects, e.g. to yield comparable horse-specific plots. Some fixed effects were removed from the horse-specific models, for instance a horse that was only ridden in light seat could not have seat included in the model.

Right lead canter was found to be associated with a higher tension than left canter, which can be seen from Figure 4 where there is a significant difference between the outside reins (or from Table 2 where the model directly states a statistical difference between left and right lead canter, but the stride cycle is not taken account of relative to this variable). However, the overall estimate difference was minor and, as the interaction with seat was not included (data not shown), this was not considered to be a main result. Corners were characterised by a lower rein tension. Half-pass to the right had a lower tension than no lateral movement and rein tension was found to increase in the order: collection < baseline < lengthening. The fact that the tension was less when the head became closer to the chest may represent loosening of rein tension when the horse's head reached a more (desired) flexed position, as a form of negative reinforcement. The other explanation is that the horse (successfully) evaded rein pressure by increasing head-neck flexion. The nose angle ROM was not linearly related to the outcome, with the next lowest category ( $\geq 15-20$ degrees) having a lower rein tension than all other categories. Albeit, as the baseline ( $<15$ degrees) had rather few data, this suggests that low ROM can be associated with a lower rein tension. 


\section{Variation}

The graphical patterns (Figure 3 and Supplementary Figure $\mathrm{S} 2$ ), suggest there are both considerable rider and horse effects and overall $26 \%$ of the variation could be attributed to the riders and $21 \%$ to the horses, which is similar to what was shown by König von Borstel and Glissman (2014). As the inside and outside rein tensions were statistically different in seven horses ridden by four riders, we suggest that this variation can be attributed to the riders to a substantial degree, also because the variable horse laterality or 'preferred bending direction' of the horse was shown to be insignificant in all rein tension models tested (data not shown). The variable handedness or laterality of riding horses was considered important as a cornerstone in riding is to be able to ride both straight and symmetrically to left and right sides (Kuhnke et al., 2010). As all riders had ridden the horses at least five times previously (in $>50 \%$ of the horses for years), it is possible that idiosyncratic cueing and response patterns had developed within each dyad. A similar study of more unfamiliar horse-rider combinations may have incurred much higher variation. However, the study does reflect habitual rein tension patterns during canter work for horses and some of the different strategies riders may use.

In order to evaluate the relevance in terms of health or welfare, more work needs to be done on the horse's performance and behavioural response to the demonstrated variation in rein tension. To achieve this, the relationship between rein tension and actual pressure on the tissues in the horse's mouth should be established and how the horse perceives this, as possibly reflected in behavioural variables. We should also strive to identify more and less optimal temporal rein tension patterns in relationship with horse kinematics and eventually performance. Rein tension should be further studied in various disciplines, in horses trained by different trainers and ridden with various types of bits. Rein tension should be studied both during every day riding and under experimental conditions in which different riders would perform specific tests riding on the same horses, to further discriminate between rider and horse effects.

\section{Limitations to the study}

There were only eight riders participating in the study and extrapolation to larger populations should be done with caution. Further, as the riders chose exercises according to their own training regime, there was low power for some exercises. Additionally, some classifications (e.g. collection and lengthening) were made by one single evaluator, which introduced an element of subjectivity. Especially for lengthening and collection there were little data, making significant findings related to these variables less likely. A GPS was used in the original set-up, but reliable data from this system were only achieved in the outdoor sessions, for which reason data on speed have not been included.

\section{Conclusions}

It is concluded that within-stride variation in rein tension during flatwork canter performed by professional dressage riders was substantial and substantially influenced by both riders and horses. In the overall analysis we found significant inside/outside rein differences, but individual variation was large, as these differences were not seen in some horserider combinations. Pressure measurements in the horse's mouth and assessment of behavioural parameters and or physiological indicators of stress level such as cortisol, seem indicated. In the future, rein tension measurements may possibly be used to assess horse obedience, rider skill and/or horse-rider match.

\section{Supplementary material}

Supplementary material can be found online at http:// dx.doi.org/10.3920/CEP150005.

Figure S1. Examples showing the variability across the underlying normalised strides and the mean normalised stride in two situations.

Figure S2. Rein tension during the stride cycle for the inside and outside rein per horse in riders 2,3 and 5-8.

\section{Acknowledgements}

The study was funded by The Swedish Research Council Formas. We thank the riders for their contributions.

\section{References}

Clayton, H.M., 1994. Comparison of the collected, working, medium and extended canters. Equine Veterinary Journal 17: 16-19.

Clayton, H.M., Larson, B., Kaiser, L.J. and Lavagnino, M., 2011. Length and elasticity of side reins affect rein tension at trot. Veterinary Journal 188: 291-294.

Clayton, H.M., Singleton, W.H., Lanovaz, J. and Cloud, G.L., 2003. Measurement of rein tension during horseback riding using strain gage transducers. Experimental Technology 27: 34-36.

Decarpentry, A., 1949. Academic equitation. A system based on the methods of D'Aure, Baucher and L'Hotte. Robert Hale Ltd, London, UK.

Egenvall, A., Tranquille, C.A., Lönnell, A.C., Bitschnau, C., Oomen, A., Hernlund, E., Montavon, S., Franko, M.A., Murray, R.C., Weishaupt, M.A., Van Weeren, R. and Roepstorff, L., 2013. Days-lost to training and competition in relation to workload in 263 elite show-jumping horses in four European countries. Preventive Veterinary Medicine 112: $387-400$. 
Eisersiö, M., 2013. How to build a rein tension meter. Degree project in biology. Swedish University of Agricultural Sciences, Uppsala, Sweden. Available at: http://tinyurl.com/lbvu8mr.

Eisersiö, M., Roepstorff, L., Rhodin, M. and Egenvall, A., 2015. A snapshot of the training schedule for 8 professional riders riding dressage. Comparative Exercise Physiology 11: 35-46.

Eisersiö, M., Roepstorff, L., Weishaupt, M.A. and Egenvall, A., 2013. Movements of the horse's mouth in relation to the horse-rider kinematic variables. Veterinary Journal 198: e33-e38.

Faber, M., Johnston, C., Schamhardt, H.C., Van Weeren, P.R., Roepstorff, L. and Barneveld, A., 2001. Three-dimensional kinematics of the equine spine during canter. Equine Veterinary Journal 33: 145-149.

Gandy, E.A., Bondi, A., Hogg, R. and Pigott, T.M.C., 2014. A preliminary investigation of the use of inertial sensing technology for the measurement of hip rotation asymmetry in horse riders. Sports Technology. DOI: http://dx.doi.org/10.1080/19346182.20 14.905949.

German National Equestrian Federation (GNEF), 1997. The principles of riding: the official instruction handbook of the German National Equestrian Federation. Kenilworth Press, Shrewsbury, UK.

Heleski, C.R., McGreevy, P.D., Kaiser, L.J., Lavagnino, M., Tans, E., Bello, N. and Clayton, H.M., 2009. Effects on behaviour and rein tension on horses ridden with and without martingales and rein inserts. Veterinary Journal 181: 56-62.

König von Borstel, U. and Glissman, C., 2014. Alternative to conventional evaluation of rideability in horse performance test: suitability of rein tension and behavioural parameters. PLoS One 9: 1-9.
Kuhnke, S., Dumbell, L., Gauly, M., Johnson, J.L., McDonald, K. and König von Borstel, U., 2010. A comparison of rein tension of the rider's dominant and non-dominant hand and the influence of the horse's laterality. Comparative Exercise Physiology 7: 57-63.

Lönnell, A.C., Bröjer, J., Nostell, K., Hernlund, E., Roepstorff, L., Tranquille, C.A., Murray, R.C., Oomen, A., Van Weeren, R., Bitschnau, C., Montavon, S., Weishaupt, M.A. and Egenvall, A., 2014. Variation in training regimens in professional showjumping yards. Equine Veterinary Journal 46: 233-238.

Lovett, T., Hodson-Tole, E. and Nankervis, K., 2004. A preliminary investigation of rider position during walk, trot and canter. Equine Comparative Exercise Physiology 2: 71-76.

McGreevy, P.D., 2007. The advent of equitation science. Veterinary Journal 174: 492-500.

Murray, R.C., Walters, J.M., Snart, H., Dyson, S.J. and Parkin, T.D.H., 2010. Identification of risk factors for lameness in dressage horses. Veterinary Journal 184: 27-36.

Symes, D. and Ellis, R., 2009. A preliminary study into rider asymmetry within equitation. Veterinary Journal 181: 34-37.

Warren-Smith, A.K., Curtis, R.A., Greetham, L. and McGreevy, P.D., 2007. Rein contact between horse and handler during specific equitation movements. Applied Animal Behaviour Science 108: 157-169.

Weishaupt, M.A., Byström, A., Von Peinen, K., Wiestner, T., Meyers, H., Waldern, N., Johnston, C., Van Weeren, R. and Roepstorff, L., 2009. Kinetics and kinematics of the passage. Equine Veterinary Journal 41: 263-267.

Xenophon, 350 BC. The art of horsemanship Available at: http:// en.wikipedia.org/wiki/On_Horsemanship. 
